\title{
Foreword: special issue for the journal track of the 8th Asian conference on machine learning (ACML 2016)
}

\author{
Robert J. Durrant ${ }^{1}$ • Kee-Eung Kim ${ }^{2}$. Geoffrey Holmes ${ }^{1}$ - Stephen Marsland ${ }^{3}$. \\ Masashi Sugiyama $^{4,5}$. Zhi-Hua Zhou'
}

Published online: 5 April 2017

(C) The Author(s) 2017

We, the guest editors, welcome you to this special issue of Machine Learning comprising papers accepted to the journal track of the 8th Asian conference on machine learning (ACML 2016), held at the University of Waikato, Hamilton, New Zealand. This year's ACML was the first to run a dedicated journal track alongside the usual proceedings track: We believe the experiment was a success and we are delighted to share these contributions with you.

This issue of Machine Learning covers a range of areas of current interest in machine learning, and these papers represent some of the state-of-the-art in our fascinating field. Unlike in previous years this ACML special issue does not contain extended versions of selected conference submissions, rather these are instead full journal papers on original

Robert J. Durrant

bobd@waikato.ac.nz

Kee-Eung Kim

kekim@cs.kaist.ac.kr

Geoffrey Holmes

geoff@waikato.ac.nz

Stephen Marsland

s.r.marsland@massey.ac.nz

Masashi Sugiyama

sugi@k.u-tokyo.ac.jp

Zhi-Hua Zhou

zhouzh@lamda.nju.edu.cn

1 University of Waikato, Hamilton, New Zealand

2 KAIST, Daejeon, Korea

3 Massey University, Palmerston North, New Zealand

4 RIKEN, Tokyo, Japan

5 The University of Tokyo, Tokyo, Japan

6 Nanjing University, Nanjing, China 
themes that were submitted to the journal track of ACML 2016 and peer-reviewed according to the usual criteria expected for this journal.

Following an open call for papers, the journal track for ACML 2016 received 28 submissions, of which the best 5 comprise this special issue.

The first article, by Qi Mao, Li Wang and Ivor Tsang: "A Unified Probabilistic Framework for Robust Manifold Learning and Embedding" focuses on the problem of learning a smooth low-dimensional representation of noisy data where, unlike earlier work on such manifold learning, complex geometry such as loops, bifurcations and disconnected components can be modelled by the authors' approach using a probabilistic framework. In particular, given a prior distribution over the noise in a set of observations and a set of inequality constraints on pairwise distances between points embedded in a manifold, the authors show how to efficiently obtain a smooth skeleton structure for the data by learning the embedding with maximum posterior probability. Extensive simulations underline the usefulness of the authors' approach.

In "Collaborative Topic Regression for Online Recommender Systems: An Online and Bayesian Approach", Chenghao Liu, Steven C.H. Hoi, Tao Jin, Peilin Zhao and Jianling Sun present a novel extension of Collaborative Topic Regression (CTR) which overcomes major limitations in the existing batch decoupled inference algorithm for CTR (bdi-CTR) (Wang and Blei, Proc. KDD 2011). In particular, unlike bdi-CTR, their approach both enables online learning of CTR while, at the same time, jointly optimizing the objectives of the probabilistic matrix factorization and topic modelling steps in the CTR algorithm. Experimentation on a real-world dataset demonstrates the authors' approach improves over bdi-CTR both in terms of RMSE on ratings prediction and compute time.

In "Progressive Random k-Labelsets for Cost-Sensitive Multi-Label Classification", YuPing $\mathrm{Wu}$ and Hsuan-Tien Lin describe an extension of a popular multi-label classification method, namely random k-labelsets, which tackles two issues with existing multi-label classification algorithms by providing an approach that on the one hand can optimise for a range of very different evaluation criteria, while at the same time having low computational time complexity. Extensive experiments confirm the authors' approach outperforms or is highly competitive with the state-of-the-art, on a wide range of metrics.

In "Non-redundant Multiple Clustering by Nonnegative Matrix Factorisation", Sen Yang and Lijun Zhang provide a new clustering algorithm which, unlike most existing algorithms, generates multiple sequential clusterings of a single dataset, while a quadratic penalty term measuring the similarity between two clusterings ensures that each new clustering is different from preceding ones. The authors' approach is evaluated against several standard measures of clustering quality on a range of datasets, while the clusterings obtained on real data do indeed capture interesting - but different - aspects of the data.

Finally in "Multi-view Kernel Completion", by Sahely Bhadra, Samuel Kaski, and Juho Rousu, the authors introduce the first algorithm that can impute entire missing rows and columns in a kernel matrix, using side information in the form of other kernel matrices which also need not be complete. Their approach works for linear and non-linear kernels. Applications of the authors' approach include combining data from multiple sources, and learning when values are missing or unobtainable (for some views) in practice. The authors demonstrate the utility of their approach on simulated data and several case studies with real-world data.

It has been an honour to serve as guest editors for this special issue, and it would not have been possible without the contributions of many people. We wish to thank all authors for their contributions to this special issue. We also would like to express our sincere gratitude to all the referees for their time and effort in ensuring the quality of the submissions for this 
issue. We also wish to thank Peter Flach, editor-in-chief for MLJ, and Dragos Margineantu, editor of special issues for MLJ, for their guidance and support, as well as Melissa Fearon and Venkat Ganesan from the Springer editorial office for their help in keeping the process running smoothly.

We hope that you enjoy this special issue as much as we have enjoyed putting it together for you, and that it provides some inspiration for your own future research. 